\title{
Corneal Involvement in Rheumatoid Arthritis: An In Vivo Confocal Study
}

\author{
Edoardo Villani, ${ }^{1}$ Daniela Galimberti, ${ }^{1}$ Francesco Viola, ${ }^{1}$ Chiara Mapelli, ${ }^{1}$ \\ Nicoletta Del Papa, ${ }^{2}$ and Roberto Ratiglia ${ }^{1}$
}

Purpose. To analyze the in vivo morphology of corneal cells and nerves in patients with rheumatoid arthritis (RA), with or without secondary Sjögren's syndrome (SSII), and to investigate the correlations between corneal alterations and RA activity.

Methods. Fifty patients with RA and 30 age- and gendermatched control subjects were studied. SSII was diagnosed according to the American-European Consensus Group criteria, and RA activity was evaluated by the Lansbury index (LI). Confocal microscopy was used to investigate corneal thickness, the number of epithelial and stromal cells, and keratocyte hyperreflectivity. In addition, the sub-basal plexus was assessed for the number, tortuosity, and reflectivity of the nerve fibers and the presence of beadlike formations.

Results. Sixteen percent of patients with RA also had SSII. Between the SSII and non-SSII groups, no significant differences were found in the LI or in the clinical and confocal variables. Significant differences were present between patients with RA and control subjects for all the variables studied except nerve reflectivity. In patients with RA with and without SSII, LI correlated significantly with the number of beadlike formations and the number of hyperreflective, activated keratocytes.

Conclusions. Confocal microscopy of patients with RA showed several changes in corneal cells and nerves. The number of beadlike formations and the number of activated keratocytes could be interpreted as confocal signs of ocular surface disease activity. These correlations with the index of systemic disease activity, LI, may provide insight regarding the pathogenic mechanisms of dry eye in patients with RA. (Invest Ophthalmol Vis Sci. 2008;49:560-564) DOI:10.1167/iovs.07-0893

$\mathbf{R}^{\mathrm{n}}$ heumatoid Arthritis (RA) is a chronic inflammatory autoimmune disease of unknown origin. It manifests itself through an erosive, symmetric synovitis and frequently has multiregional, extra-articular manifestations. Its course is chronic, recurring in most cases with quiescent phases alternating with relapses., ${ }^{1,2}$ The eye, and the ocular surface in

From the ${ }^{1}$ Clinica Oculistica Università degli Studi di Milano, Fondazione Ospedale Maggiore Policlinico, Mangiagalli e Regina Elena, IRCCS (Istituto Ricerca e Cura a Carattere Scientifico), Milan, Italy; and ${ }^{2}$ Dipartimento di Reumatologia Ospedale Gaetano Pini, Milan, Italy.

Presented in part at the annual meeting of the Association for Research in Vision and Ophthalmology, Fort Lauderdale, Florida, May 2007.

Submitted for publication July 16, 2007; revised October 2, 2007; accepted December 19, 2007.

Disclosure: E. Villani, None; D. Galimberti, None; F. Viola, None; C. Mapelli, None; N. Del Papa, None; R. Ratiglia, None

The publication costs of this article were defrayed in part by page charge payment. This article must therefore be marked "advertisement" in accordance with 18 U.S.C. $\$ 1734$ solely to indicate this fact.

Corresponding author: Edoardo Villani, Clinica Oculistica Università degli Studi di Milano, Fondazione Ospedale Maggiore Policlinico, Mangiagalli e Regina Elena, IRCCS, Via Francesco Sforza 35, 20122 Milan, Italy; eddy.villani@tiscali.it. particular, is one of the regions frequently affected by this disease. ${ }^{3,4}$ The most frequent eye complication of RA, though not the most severe one, is keratoconjunctivitis sicca (KCS). With extended criteria for the diagnosis of dry eye, ${ }^{5}$ the prevalence in RA is approximately $90 \%$.

Approximately $11 \%$ to $31 \%$ of persons with RA have secondary Sjögren's syndrome (SSII). One of the main clinical signs of SSII, as well as of primary Sjögren's syndrome, is KCS. ${ }^{6}$ The dry eye associated with Sjögren's syndrome is viewed as a prototype not only of the KCS associated with autoimmune diseases, but also of the hyposecretory dry eye in general. ${ }^{7,8}$

In this study we used confocal microscopy to analyze the corneal morphologic alterations in patients with RA, with or without SSII. We evaluated potential correlations between ocular surface manifestations of RA and the activity of systemic RA. These correlations are particularly interesting, because recent reports suggest that there are profound etiologic and pathogenic differences between KCS in patients with RA with SSII and patients with RA without SSII, though they lack manifest differences in the clinical indicators. ${ }^{5,9}$ Confocal microscopy appears to be an essential instrument for exploring the nature of corneal alterations in such patients, as it provides quick, noninvasive, in vivo microstructural evaluations.

\section{Methods}

\section{Patients}

Fifty patients with RA were consecutively recruited ( 46 women and 4 men; average age, $52 \pm 13.1$ years) after referral to our center by rheumatologists. In all patients, the possible presence of SSII was investigated in accordance with the American-European Consensus Group criteria. ${ }^{10}$ These criteria require the presence of ocular or oral symptoms and the presence of at least two of the following three variables: ocular signs, histopathology in minor salivary glands, and objective evidence of salivary gland involvement. The Lansbury index (LI) ${ }^{11,12}$ was calculated to rate the degree of systemic RA activity. The control group (C) included 30 healthy people ( 24 women and 6 men; average age of $50.4 \pm 21.2$ years) attending our general clinic.

The following were considered exclusion criteria: the presence of lymphomas, AIDS, sarcoidosis, or diabetes mellitus; dystrophies or inflammations of the ocular surface; systemic treatments with drugs with known corneal toxicity; local treatments with drugs against glaucoma and steroidal or nonsteroidal anti-inflammatory drugs (NSAIDs); the use of contact lenses; and previous ophthalmic surgery. We also excluded from the study those patients for whom the rheumatologist in charge of the case expressed a negative opinion on the execution of the anamnestic, clinical, and laboratory evaluations necessary to quantify the LI and the possible diagnosis of SSII.

All participants signed an informed consent. The study was conducted in accordance with the guidelines of the Helsinki Declaration and the Italian privacy law.

\section{Clinical Evaluation}

An accurate medical history was drawn up for each participant in the study, and all patients completed a questionnaire for a standardized evaluation of dry eye-related symptoms (Ocular Surface Disease Index; 
TABLE 1. Clinical Data

\begin{tabular}{lcccccc}
\hline & Questionnaire & BUT & Schirmer I & Sensitivity & Fluo & LI \\
\hline RA with SS & $2.39 \pm 0.53$ & $3.60 \pm 1.68$ & $4.25 \pm 2.41$ & $4.93 \pm 0.25$ & $2.43 \pm 0.90$ & $39.99 \pm 12.95$ \\
RA without SS & $2.29 \pm 0.81$ & $3.70 \pm 1.90$ & $4.71 \pm 3.23$ & $5.03 \pm 0.52$ & $2.10 \pm 1.24$ & $34.45 \pm 15.64$ \\
Control & $0.52 \pm 0.56$ & $10.47 \pm 1.83$ & $14.83 \pm 3.47$ & $5.85 \pm 0.38$ & - & - \\
$P$ & $<0.001^{*}$ & $<0.001 \dagger$ & $<0.001 \neq$ & $<0.001 \Phi$ & 0.479 & 0.352 \\
& & & &
\end{tabular}

$P$ is by ANOVA.

${ }^{*}$ RA with SS vs. C, RA without SS vs. C; $P<0.001$, LSD. RA with SS vs. RA without SS; $P=0.586$, LSD.

$\dagger$ RA with SS vs. C, RA without SS vs. C; $P<0.001$, LSD. RA with SS vs. RA without SS; $P=0.806$, LSD

$\ddagger$ RA with SS vs. C, RA without SS vs. C; $P<0.001$, LSD. RA with SS vs. RA without SS; $P=0.716$, LSD.

$\S$ RA with SS vs. C, RA without SS vs. C; $P<0.001$, LSD. RA with SS vs. RA without SS; $P=0.584$, LSD.

OSDI). ${ }^{13}$ All the subjects included in the study underwent a thorough ophthalmic evaluation, including biomicroscopic examination of ocular adnexa and anterior segment, evaluation of the breakup time of the tear film (BUT), tear secretion (Schirmer test with local anesthetic), corneal apex sensitivity (Cochet-Bonnet nylon thread aesthesiometer), and corneal surface staining with sodium fluorescein $1 \%$ collyrium, scored according to the van Bijsterveld method. ${ }^{14}$ Both eyes were examined in all subjects. For statistical analysis of clinical and confocal data, the eye recording the highest score with the van Bijsterveld fluorescein test was selected. In case of equal score for the two eyes, the discriminant criteria considered were, by order of relevance, the lower corneal sensitivity and the lower score on the Schirmer test.

\section{Confocal Microscopy}

Image Acquisition. The examination with the confocal microscope (Confoscan 2.0; Nidek Technologies, Vigonza, Italy) was conducted in an area of $440 \times 330 \mu \mathrm{m}$, approximately at the corneal apex, according to an already published procedure. ${ }^{15}$

Image Analysis. The scanned confocal images were analyzed by the same masked investigator (EV). According to a previously described method, ${ }^{15}$ we quantified the corneal and stromal thicknesses, the cell densities of the superficial and basal epithelium, and the cell densities of the anterior and posterior stroma. We quantified the number of the sub-basal plexus nerve fibers visible in one frame and

TABLE 2. Corneal and Stromal Thicknesses: Confocal Data

\begin{tabular}{lcc}
\hline & Corneal Thickness & Stromal Thickness \\
\hline RA with SS & $514.75 \pm 17.14$ & $467.87 \pm 17.57$ \\
RA without SS & $521.02 \pm 21.45$ & $475.90 \pm 19.82$ \\
Control & $559.23 \pm 24.46$ & $499.43 \pm 25.38$ \\
$P$ & $<0.001^{*}$ & $<0.001 \dagger$ \\
\hline
\end{tabular}

\section{$P$ is by ANOVA.}

${ }^{*}$ RA with SS vs. C, RA without SS vs. C; $P<0.001$, LSD. RA with SS vs. RA without SS; $P=0.468$, LSD.

† RA with SS vs. C, RA without SS vs. C; $P<0.001$, LSD. RA with SS vs. RA without SS; $P=0.345$, LSD. the tortuosity and reflectivity of those fibers. ${ }^{16}$ We also counted the number of beadlike formations per $100 \mu \mathrm{m}$ of nerve fiber, as described by Benitez del Castillo et al. ${ }^{7}$ Finally, we quantified the number of activated keratocytes, defined as the highest number of hyperreflective keratocytes visible in one frame of stroma. A second independent investigator analyzed the images, quantifying the cell density in the different layers, the number of activated keratocytes, and the number of beadlike formations. These data were used to calculate the interobserver variance.

\section{Statistical Analysis}

All data are expressed as the mean $\pm \mathrm{SD}$. The normal distribution of the variables was verified through the Kolmogorov-Smirnov test. Comparisons among the mean scores were made through one-way ANOVA with least-significant difference (LSD) post hoc test. The correlations among variables were studied with Spearman's index of linear correlation. The minimum criterion for tests of significance was $P<0.05$. The statistical analysis was conducted with commercial software (SPSS for Windows, ver. 12.0; SPSS Sciences, Chicago, IL).

\section{Results}

\section{Clinical Data}

Eight (16\%) of the 50 patients with RA had SSII. For the clinical data derived from the OSDI questionnaire, the scores for the patients with RA with SSII and those without it were significantly greater than the control subjects $(P<0.001$, Table 1$)$. For BUT, Schirmer test, and corneal sensitivity, the scores for patients with RA with and without SSII were significantly less than those of the control subjects $(P<0.001)$. None of the variables analyzed were significantly different between the two RA groups.

\section{Pachymetry and Cell Density Data}

Each observation by confocal microscopy took 1.5 to 2.5 minutes, and none of the participants showed any relevant alteration of the ocular surface as a result of the examination. Both

TABLE 3. Cell Densities and Number of Activated Keratocytes: Confocal Data

\begin{tabular}{|c|c|c|c|c|c|}
\hline & Superficial Epithelium & Basal Epithelium & Anterior Stroma & Posterior Stroma & Activated Keratocytes \\
\hline RA with SS & $993.11 \pm 104.82$ & $5980.32 \pm 192.58$ & $1226.50 \pm 69.78$ & $835.50 \pm 64.67$ & $7.02 \pm 3.27$ \\
\hline RA without SS & $1024.92 \pm 183.02$ & $5820.69 \pm 221.40$ & $1190.88 \pm 90.97$ & $800.78 \pm 116.08$ & $5.89 \pm 4.08$ \\
\hline Control & $1511.60 \pm 130.95$ & $5602.23 \pm 234.89$ & $970.33 \pm 104.47$ & $702.23 \pm 79.22$ & $0.45 \pm 0.66$ \\
\hline$P$ & $<0.001^{*}$ & $<0.001 \dagger$ & $<0.001 \ddagger$ & $<0.001 \oint$ & $<0.001 \|$ \\
\hline
\end{tabular}

$P$ is by ANOVA.

${ }^{*}$ RA with SS vs. C, RA without SS vs. C; $P<0.001$, LSD. RA with SS vs. RA without SS; $P=0.610$, LSD.

† RA with SS vs. C, RA without SS vs. C; $P<0.001$, LSD. RA with SS vs. RA without SS; $P=0.068$, LSD

‡ RA with SS vs. C, RA without SS vs. C; $P<0.001$, LSD. RA with SS vs. RA without SS; $P=0.332$, LSD.

$\S$ RA with SS vs. C, RA without SS vs. C; $P<0.001$, LSD. RA with SS vs. RA without SS; $P=0.369$, LSD.

$\|$ RA with SS vs. C, RA without SS vs. C; $P<0.001$, LSD. RA with SS vs. RA without SS; $P=0.355$, LSD. 


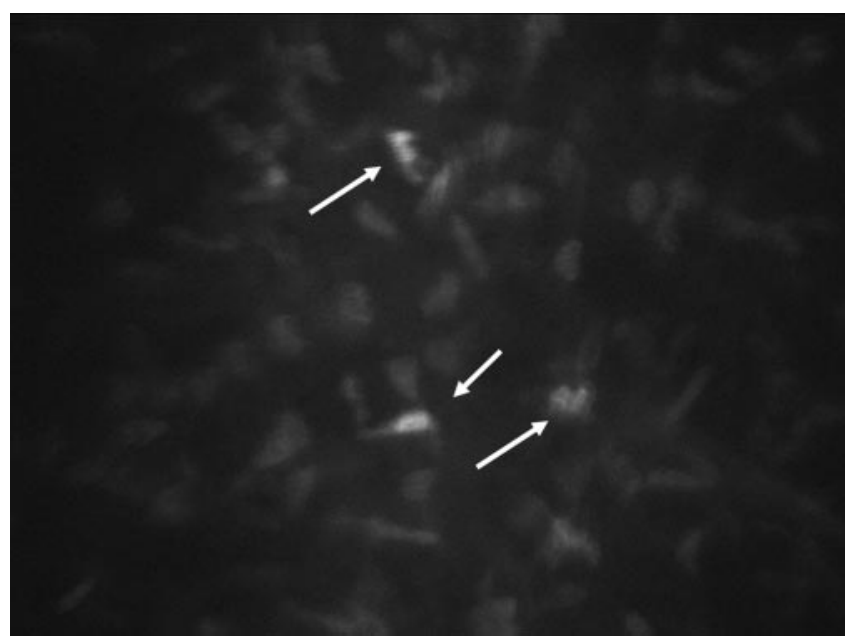

FIGURE 1. The activated keratocytes shown are hyperreflective stromal cells.

the cornea and stroma were significantly thinner in patients with RA than in healthy subjects $(P<0.001$, Table 2$)$. However, there were no differences between the two RA groups. The density of cells (per square millimeter) in the superficial epithelium, basal epithelium, anterior stroma, and posterior stroma of the RA groups with or without SSII were not significantly different from one another (Table 3). Likewise, the number of hyperreflective activated keratocytes (Fig. 1) was similar in both RA groups. In comparison to control subjects, the cell density of the superficial epithelium in both RA groups was significantly lower $(P<0.001)$. In contrast, the cell densities of the basal epithelium, anterior stroma, and posterior were all significantly greater than those of the control group $(P<0.001)$. Similarly, the number of activated keratocytes in the both RA groups was greater than in the control subjects $(P<0.001)$. The interobserver variance was $3 \%, 2 \%, 5 \%$, and $5 \%$ for the cell densities of the superficial epithelium, the basal epithelium, the anterior stroma, and the posterior stroma, respectively. It was $22 \%$ for the number of activated keratocytes. There was strong correlation between the values obtained by the two investigators for all variables $(P<0.001$; Spearman).

\section{Sub-basal Plexus Data}

Within the sub-basal nerve plexus, the number of nerve fibers, tortuosity, reflectivity, and beadlike formations were similar among both RA groups (Table 4). However, the number of nerves in the RA groups was significantly less than in the control group, whereas the tortuosity and number of beadlike formations (Fig. 2) was significantly greater $(P<0.001)$. The reflectivity was similar between the RA and control groups. For beadlike formations, the interobserver variance was $8 \%$, with a strong correlation between the values obtained by the two investigators $(P<0.001$, Spearman).

\section{Correlations of Clinical and Confocal Data}

There was a statistically significant correlation between the LI and the Schirmer test for all patients with RA $(P<0.05$, Spearman). This correlation was evident for the RA with SSII group $(P<0.05)$; however, it was not significant for the RA without SSII group $(P=0.065)$. The LI also correlated with the number of beadlike formations and the number of activated keratocytes in the RA group with SSII $(P<0.01$ and $P<0.05$ respectively; Figs. 3A, 3B). Similar correlations were also present in the RA group without SSII $(P<0.05$ and $P<0.05$ respectively; Figs. 3C, 3D).

The patients with RA, both with and without SSII, showed several significant correlations among the clinical and confocal variables. The main ones are reported in Table 5.

\section{Discussion}

In the past few years, confocal microscopy has offered new opportunities to study the in vivo structure of the cornea. The technique permits visualization of disease-related microstructural alterations in the cornea by means of a noninvasive examination that is quick to execute and repeatable. According to the model used in recent studies, we used confocal microscopy in a quantitative way to evaluate RA-related changes in the cornea. In our opinion, this approach represents an important evolution in the use and application of this technology.

As in a previous study of patients with Sjögren's syndrome, ${ }^{15}$ we found a surprising association between the standardized OSDI evaluation of symptoms and some clinical signs of the ocular surface. Many articles in literature emphasize the scant association between signs and symptoms in dry eye,${ }^{17-19}$ whereas others report occasional associations ${ }^{8,20}$ or inverse correlations. ${ }^{21}$ Such heterogeneous data are probably due to the small size of the samples used in many of those studies and to the characteristics of the different questionnaires used.

Both groups of our patients with RA had central corneal and stromal thicknesses that were significantly thinner than the control group. These changes also occur for KCS in general ${ }^{22}$ and for KCS combined with autoimmune disease. ${ }^{15,23}$ The thinning could occur by increased apoptotic and proteolytic phenomena of the stroma. Thinning may also be the result of increased tangential forces that act on the epithelial surface which can be abnormally irregular.

The superficial and basal epithelial cell densities and the apparent $^{24}$ anterior and posterior stromal cell densities in pa-

TABLE 4. Number and Morphology of Subbasal Nerves: Confocal Data

\begin{tabular}{lcccc}
\hline & Nerve Number & Tortuosity & Reflectivity & Beadlike Formations \\
\hline RA with SS & $3.18 \pm 0.74$ & $2.65 \pm 0.47$ & $1.99 \pm 0.70$ & $332.94 \pm 64.20$ \\
RA without SS & $3.32 \pm 1.14$ & $2.53 \pm 0.93$ & $2.15 \pm 1.38$ & $307.26 \pm 78.81$ \\
Control & $4.99 \pm 0.81$ & $1.33 \pm 0.60$ & $2.02 \pm 1.01$ & $196.16 \pm 0.66$ \\
$P$ & $<0.001^{*}$ & $<0.001 \dagger$ & 0.874 & $<0.001 \neq$ \\
\hline
\end{tabular}

\footnotetext{
LSD.

$P$ is by ANOVA.
${ }^{*}$ RA with SS vs. C, RA without SS vs. C; $P<0.001$, LSD. RA with SS vs. RA without SS; $P=0.708$,

† RA with SS vs. C, RA without SS vs. C; $P<0.001$, LSD. RA with SS vs. RA without SS; $P=0.699$, LSD. LSD.

‡ RA with SS vs. C, RA without SS vs. C; $P<0.001$, LSD. RA with SS vs. RA without SS; $P=0.373$,
} 
Figure 2. Sub-basal nerve fibers (A), with evident beadlike formations (B, arrows).
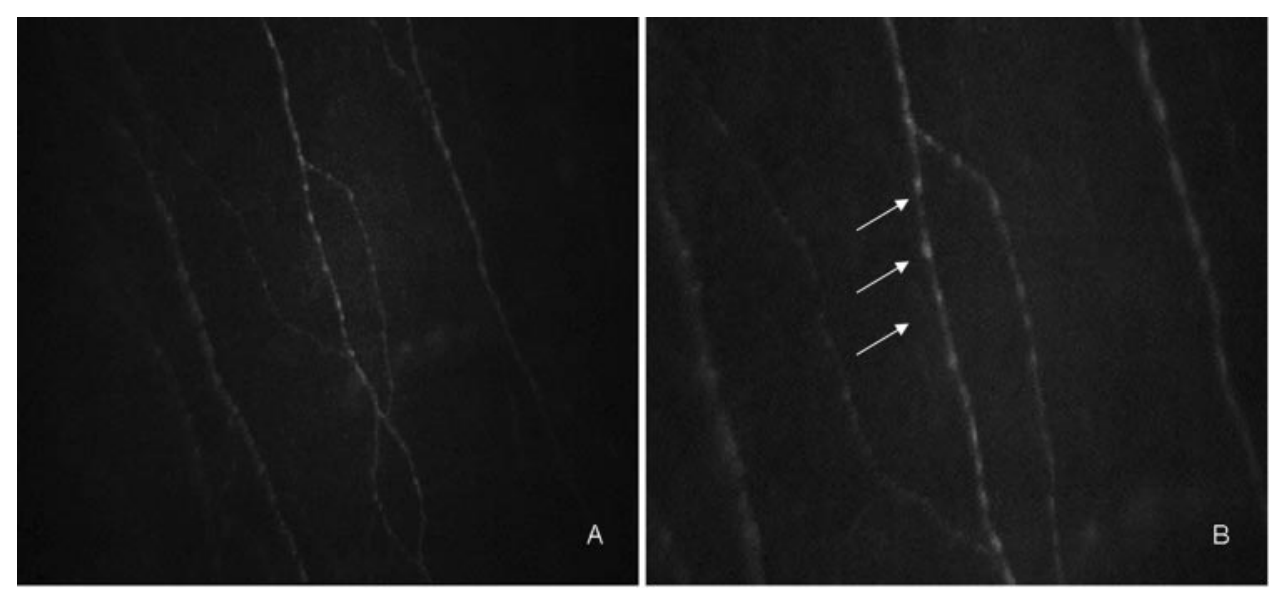

tients with RA with SSII are similar to those previously reported. ${ }^{15}$ We also found similar values in patients with RA without SSII.

For patients in both RA groups, we observed a significantly higher number of hyperreflective stromal cells than in the control group. These keratocytes are in a specific stage of metabolic activation induced by proinflammatory cytokines, such as IL-1 and IL-6. Benitez del Castillo et al. ${ }^{8}$ have recently discussed the key role played by those keratocytes in the inflammatory process that is linked to dry eye-related disease.
As a matter of fact, the activated keratocytes are seemingly able to produce nerve growth factor, thus making a relevant contribution to the processes of activation and reorganization of the nerve fibers of the sub-basal plexus. In both groups of patients with RA, the nerve fibers showed increased tortuosity and a higher number of beadlike formations, which could be signs of metabolic activation of the plexus. ${ }^{25}$ This is consistent with the correlations registered between the LI, an index of activity of the systemic disease, and the number of activated keratocytes and the number of beadlike formations in all pa-
FIGURE 3. Correlations with the LI. The LI index in the patients with RA with SSII correlated significantly with (A) the number of beadlike formations $(r=0.881 ; P<0.01)$ and (B) the number of activated keratocytes $(r=0.714 ; P<0.01)$. In patients with RA without SSII, similar correlations were evident for LI and (C) the number of beadlike formations $(r=0.383 ; P<0.05)$ and $(\mathbf{D})$ activated keratocytes $(r=0.342 ; P<$ $0.05)$.
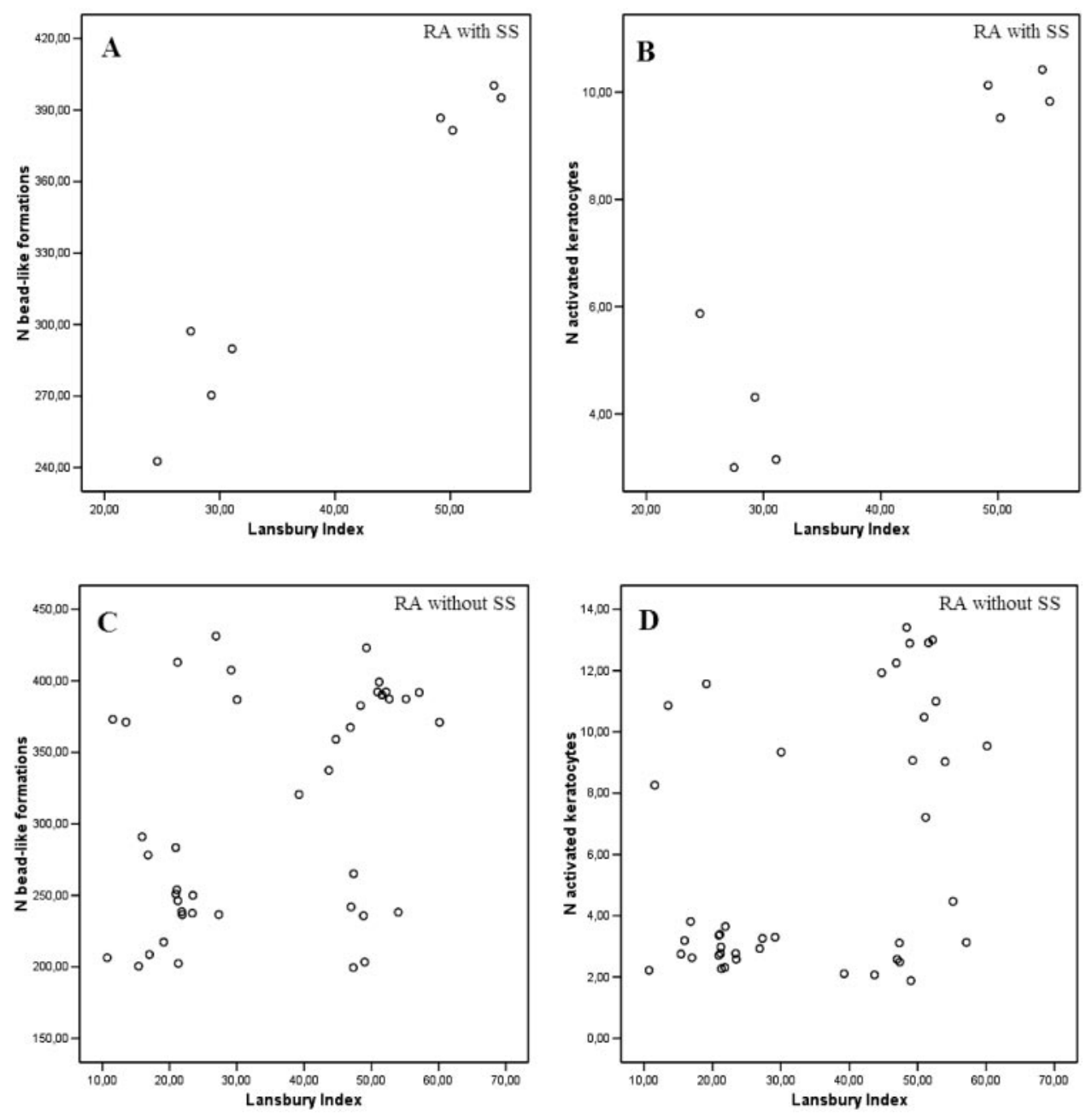
TABle 5. Principal Correlations among Variables

$\boldsymbol{P}$

(RA with SSII)

\begin{tabular}{lll}
\hline Questionnaire & & \\
Schirmer & $<0.01$ & $<0.01$ \\
Fluo & $<0.01$ & $<0.01$ \\
Sensitivity & $<0.01$ & $<0.01$ \\
Tortuosity & $<0.01$ & $<0.01$ \\
Schirmer & $<0.01$ & $<0.001$ \\
$\quad$ Superficial epithelium & $<0.01$ & $<0.001$ \\
Basal epithelium & $=0.01$ & $<0.01$ \\
Tortuosity & $<0.01$ & $<0.01$ \\
$\quad$ Number of nerves & $<0.01$ & $<0.001$ \\
Fluo & $<0.01$ & $<0.001$ \\
Superficial epithelium & $<0.01$ & $<0.01$ \\
Basal epithelium & $<0.01$ & $<0.05$ \\
Tortuosity & & $<0.001$ \\
Number of nerves & $<0.05$ & $=0.074$ \\
Sensitivity & $<0.01$ & \\
Tortuosity & & \\
Number of nerves & & \\
\hline
\end{tabular}

$P$ is by Spearman linear correlation index.

tients with RA. In a recent study, Fujita et al. ${ }^{5}$ explored the possible correlations between RA activity and the alterations of the ocular surface. They reported a significant association between clinical tests for the ocular surface and the systemic disease only in patients who have RA with SSII. The validity and repeatability of the correlations between the systemic disease activity and the number of activated keratocytes and of beadlike formations should be carefully verified in broader studies, possibly conducted in a prospective way. Therefore, they cannot be used at the moment to draw conclusions on the pathogenic processes.

There were no differences in any of the variables that we evaluated when patients with RA with SSII were compared with patients with RA without SSII. These results are similar to previous findings obtained comparing patient groups with primary and secondary Sjögren's syndrome ${ }^{15}$ and suggest that the same kinds of inflammatory and neurodegenerative and regenerative changes occur in the different autoimmune types of dry eye. These alterations show various intensities and associations with the severity of both the ocular surface and the systemic disease that are worthy of further studies.

In conclusion, confocal microscopy enabled us to identify several alterations of cell activity and innervation of the cornea in patients with RA with and without SSII. The potential of confocal microscopy has not yet been fully investigated for the study of pathologic processes and the complex interaction between the ocular surface and autoimmune systemic diseases. Our results are a stimulus for further development of new approaches to confocal microscopy in patients with KCS and autoimmune diseases. This approach may lend itself to the identification of some indexes of disease activity on the ocular surface that could be compared with the indexes of activity of the systemic disease.

\section{References}

1. Grassi W, De Angelis R, Lamanna G, Cervini C. The clinical features of rheumatoid arthritis. Eur J Radiol. 1998;27(suppl 1):S18-S24.

2. Scutellari PN, Orzincolo C. Rheumatoid arthritis: sequences. Eur J Radiol. 1998;27(suppl 1):S31-S38.
3. Robin J, Dugel R. Immunologic Disorders of the Cornea and Conjunctiva. New York: Churchill Livingstone; 1988:533-537.

4. Jabs D. Ocular Manifestations of the Rbeumatic Diseases. Philadelphia: JB Lippincott; 1992;1-5.

5. Fujita M, Igarashi T, Kurai T, et al. Correlation between dry eye and rheumatoid arthritis activity. Am J Opbthalmol. 2005;140:808813.

6. Fox RI, Stern M, Michelson P. Update in Sjogren syndrome. Curr Opin Rbeum. 2000;12:391-398.

7. Benitez del Castillo JM, Wasfy MA, Fernandez C, Garcia-Sanchez J. An in vivo confocal masked study on corneal epithelium and sub-basal nerves in patients with dry eye. Invest Opbthalmol Vis Sci. 2004; 45:3030-3035.

8. Benitez del Castillo JM, Acosta MC, Wassfi MA, et al. Relation between corneal innervation with confocal microscopy and corneal sensitivity with noncontact esthesiometry in patients with dry eye. Invest Ophthalmol Vis Sci. 2007;48:173-181.

9. Lemp MA. Dry eye (keratoconjunctivitis sicca), rheumatoid arthritis, and Sjogren's syndrome. Am J Opbthalmol. 2005;140:898899.

10. Vitali C, Bombardieri S, Jonsson R, et al. Classification criteria for Sjögren's syndrome: a revised version of the European criteria proposed by the American-European Consensus Group. Ann Rbeum Dis. 2002;61:554-558.

11. Lansbury J. Quantitation of the activity of rheumatoid arthritis, I: a method for recording its systemic manifestations. Am J Med Sci. 1956;231:616-621.

12. Lansbury J. Numerical method of evaluating the status of rheumatoid arthritis. Ann Rbeum Dis. 1958;17:101-107.

13. Schiffman RM, Christianson MD, Jacobsen G, Hirsch JD, Reis BL. Reliability and validity of the Ocular Surface Disease Index. Arch Ophthalmol. 2000;118:615-621.

14. van Bijsterveld OP. Diagnostic tests in the sicca syndrome. Arch Opbthalmol. 1969;82:10-14.

15. Villani E, Galimberti D, Viola F, Mapelli C, Ratiglia R. The cornea in Sjögren's syndrome: an in vivo confocal study. Invest Ophthalmol Vis Sci. 2007;48:2017-2022.

16. Oliveira-Soto L, Efron N. Morphology of corneal nerves using confocal microscopy. Cornea. 2001;20:374-384.

17. Schein OD, Tielsch JM, Munoz B, Bandeen-Roche K, West S. Relation between signs and symptoms of dry eye in the elderly: a population-based perspective. Opbthalmology. 1997;104:13951401.

18. Hay EM, Thomas E, Pal B, Hajeer A, Chambers H, Silman AJ. Weak association between subjective symptoms or and objective testing for dry eyes and dry mouth: results from a population based study. Ann Rbeum Dis. 1998;57:20-24.

19. Nichols KK, Nichols JJ, Mitchell GL. The lack of association between sign and symptoms in patients with dry eye disease. Cornea. 2004;23:762-770.

20. Bjerrum KB. Test and symptoms in keratoconjunctivitis sicca and their correlation. Acta Opbtbalmol Scand. 1996;74:436-441.

21. Adatia FA, Michaeli-Cohen A, Naor J, Caffery B, Bookman A, Slomovic A. Correlation between corneal sensitivity, subjective dry eye symptoms and corneal staining in Sjögren's syndrome. Can J Ophthalmol. 2004;39:767-771.

22. Liu Z, Pflugfelder SC. Corneal thickness is reduced in dry eye. Cornea. 1999;18:403-407.

23. Tuominen IS, Konttinen YT, Vesaluoma MH, Moilanen JA, Helinto M, Tervo TM. Corneal innervation and morphology in primary Sjögren's syndrome. Invest Opbthalmol Vis Sci. 2003;44:25452549.

24. Patel SV, McLaren JW, Hodge DO, Bourne WM. Normal human keratocyte density and corneal thickness measurement by using confocal microscopy in vivo. Invest Ophthalmol Vis Sci. 2001;42: 333-339.

25. Zhang M, Chen J, Luo L, Xiao Q, Sun M, Liu Z. Altered corneal nerves in aqueous tear deficiency viewed by in vivo confocal microscopy. Cornea. 2005;24:818-824. 